\title{
Nurses' Attitudes Related To Alarm Fatigue in Critical Care Units: A Systematic Review
}

\author{
Mohammad Salous ${ }^{1}$, Jafar Alkhawaldeh ${ }^{2}$, Sadeq Kewan ${ }^{3}$, Hafez Aburashideh ${ }^{4}$, \\ Dania Bni Hani ${ }^{5}$, Abdulkarim Alzayyat ${ }^{6}$ \\ ${ }^{I}$ (critical care nurse, RN, MSC, quality Coordinator/Alkhalidi hospital, Amman ,Jordan) \\ ${ }^{2}$ (critical care nurse, RN, MSC/ Ph.D. practitioner student, UPM University, Amman, Jordan) \\ ${ }^{3}$ (critical care nurse, RN, MSN/ Clinical instructor, Arab medical center, Amman, Jordan) \\ ${ }^{4}(\mathrm{RN}, \mathrm{MAN}, \mathrm{CPHQ} / \mathrm{Quality}$ coordinator,Al khalidi hospital, Amman, Jordan) \\ 5 (student nurse, the university of Jordan, Amman, Jordan) \\ ${ }^{6}($ RN, MSC, Ph.D student , university of Florida, Florida, USA)
}

\begin{abstract}
Critical care unit is a place where clinical alarms occur frequently, of which many are false alarms, which leads to what is called 'alarm fatigue'. Alarm Fatigue occurs when nursing staff are exposed to a large number of false alarms which can cause alarm desensitization. Alarm fatigue in ICU was found to be an international problem and the number one medical technology related hazard. therefore the purpose of this review was to examine the attitudes of critical care nurses related to alarm fatigue.
\end{abstract}

Keywords: Alarm fatigue, clinical alarm, false alarms, noise alarms, patient safety.

\section{Introduction}

The review of related literature in this chapter was done using electronic research from databases; PubMed, CINAHL, Medline, and Google scholar. Each database was searched using the following keywords: Alarm fatigue, clinical alarm, noise alarms, patient safety, and false alarms. The Articles chosen included those published from 2002 - 2016 as well as those with information from historical background related to the alarm fatigue and its effect on patient safety. A total of 120 articles were found using the key words in the selected search engines, of which 50 were found to be useful to support the study.

\subsection{Theoretical definition of related concepts:}

\subsubsection{Intensive and Critical care units (ICCU)}

Florence Nightingale in the 1800s stated that the patients post major surgery must be treated in a separate area of the hospital [16]. In the 1900s, the John Hopkins Hospital in Baltimore opened a three-bed intensive care unit for postoperative neurosurgical, this was soon followed in Chicago, where a premature infant unit was established [16].The societal issues have affected the improvement of critical care as a department. During World War II, shock wards to care for seriously injured patients were founded [34]. The lack of nurses after the war raised the need for grouping of postoperative patients to designated recovery areas to be provided with the necessary care and monitoring their health status [34] .Further wars of the $20^{\text {th }}$ century led to improvement in the technology and experience of health care providers, which provide space for specialization in medical and nursing fields globally [34] . In 1950s, there was a booming innovation of new medical machines that help saving lives and support critically ill patients, such as; mechanical ventilator, invasive blood pressure (IBP), and intracranial pressure (ICP) monitoring. These devices communicate the changes in status of the patients to the staff by alarm signals descript by clinical alarms [34].The critical care developed from the recognition that the needs of acute and chronic ill patients, and life-threatening patients could be better satisfied if critical patients were transferred to specialized units in the hospital [34].

Critical care unit can be defined as a place where patients with advanced medical conditions such as cardiac diseases, gastrointestinal diseases, renal diseases, sepsis, and chronic respiratory illnesses can be monitored in a well-established system and advanced machines [15] .

\subsubsection{Clinical alarms}

The clinical alarms are used to enhance safety by alerting care provider to any deviations from normal status. The alarms alert care provider when there is a deterioration in patient's condition and there is a need for intervention, or when a device is not working as it should [30].

\subsubsection{Alarm fatigue (AF)}

Alarm fatigue is a problem resulting from desensitization and sensory overload; due to excessive number of alarms, which leads to lack to response among critical care nurses [9] .

\begin{tabular}{lll}
\hline DOI: $10.9790 / 1959-0602076266$ & www.iosrjournals.org & $62 \mid$ Page
\end{tabular}


When the healthcare provider is exposed to a huge number of alarms, many of which are false alarms, within working time, they will suffer from sensory overload which leads to ignoring or delaying in response to the alarm [18] . This can cause harm to the patient indirectly, and place patient at risk of severe injury or death [17].

\subsection{Operational definition of the terms:}

The operational definition specifies the way each term or concept is to be used in the study including the methods by which each will be derived or measured and their level of measurement, if applicable. To facilitate agreement on the terminologies that were used in the study, the following are operationally defined:

Critical Care Unit (CCU): area within a hospital that caters to the care of patients with critical and lifethreatening conditions; in this study is of three types: medical for cases that do not demand surgical interventions, surgical for cases that required major surgeries, and mixed (medical/surgical) for cases that might be either medical or surgical in nature.

Public hospitals: refers to the two governmental hospitals that participated in the study which are partially owned and funded by the government for which services are provided at low charges compared to other nongovernmental hospitals.

Private hospitals: refers to the four hospitals that participated in the study which are owned by a for-profit corporation and privately funded through payment of medical services by patients, insurers or foreign embassies.

Marital status: refers to civil status of the participants; indicated as either "single" or "married" and classified as nominal data.

Length of experience: refers to the actual number of years the participant has worked in the ICU; measured as interval level data.

Attitudes toward Clinical Alarms: refers to the second part of the tool including 20 items five- point Likert scale from the clinical alarm survey version 2011.

\subsection{Development of clinical alarms:}

The first problem of alarms was first brought into attention in 1974, when the published sentinel event report related to alarm by Emergency Care Research Institute (ECRI) declared that an ignored alarm on a hypothermia machine resulted in patient's burns, the method of alarming in the hypothermia machine was through a light that flashes continuously when patient is in critical condition [10]. Because this way of alarming was not effective and led to serious damage to the patient; as the alarm was ignored or not even noticed, this led the researchers to find better ways for alarming. In 1982, researchers recognized that there is a need to add sound alarms rather than the old visual alarms only (Stafford 1982)Later, Kerr and Hayes (1983)mentioned that every patient must have six or more alarms to effectively call attention of the healthcare provider with regards to the serious patient's condition. In 1999, there were many types of alarms including 40 different alarms for commonly used machines such as the electrocardiogram (ECG), ventilator, pulse oximetry, and arterial blood pressure [7]

Findings of several studies supported the argument that most of clinical alarms are actually false alarms and not related to patient condition.Sendelbach and Funk (2013) found that as many as $72 \%$ to $99.4 \%$ of clinical alarms were false alarms. Further, the excessive number of alarms has significantly led to increase fatigability among healthcare providers [30]. Lawless (1994), conducted a study in pediatric ICU settings, the results exhibited that more than $94 \%$ of alarm sounds were not true alarms; $68 \%$ of the alarms were false, and in another $26.5 \%$ patient conditions did not need emergent intervention, while only5.5\% of alarms were actually emergency situations. Authority (2008)retrieved277 incident reports related to clinical alarm, three of which were dramatically sentinel and ended with patients' death. The state of Pennsylvania in 2008 emphasized the importance of focusing on clinical alarms in an effort to decrease alarms related death and major injuries [1] .

A patient was sent to abdominal surgery, wherein the alarms were switched off intraoperatively ,then the staff forgot to turn on again, which resulted in unnoticed acute respiratory distress, eventually the patient died due to related complications after 11 days [30].In another case, a 17 years old patient died after a tonsillectomy operation. The patient suffered from respiratory distress and low oxygen saturation without attention of the healthcare providers; because the oxygen monitor alarm was silent [29] .

Because of the catastrophic outcome in many cases because of the inactive alarm systems, the Joint Commission set expectations that by 2016 that all accredited hospitals will establish and put into action policies and procedures for managing alarms, as well as they need to educate their staff, especially those in direct contact with alarm systems, about the purpose and proper operation of alarms systems for which they are responsible [30] . 


\subsection{Health care provider's responses to clinical alarms:}

The staff in CCU are expected to provide attention and respond immediately to any changes in patients 'conditions. The use of sophisticated machines which is designated to alarm for serious changes in patients' status in these units is common [6] .Therefore, the number and frequency of false alarms in critical areas may lead to lack of trust in the alarm sound, diminished response from health care provider and alarm desensitization [9] .Bustamante, Bliss et al. (2007) conducted a study to investigate the effect of having different thresholds of alarm systems and staff workload on the healthcare providers' response to alarms. The threshold of the alarm system was manipulated by changing the value along the Receiver Operation Characteristic (ROC) [2], the ROC curve was selected to reflect the sensitivity of the alarm system. The study used experimental design, with a sample of 132 participants from Dominion University in United State of America. The results of the study showed faster response to true alarms from the participants when they have less workload and using a higher thresholds of alarms.

In addition, the nurses' response to clinical alarms depends on other factors; like personal evaluation to the urgency of the alarm based on the patient's condition. The work overload and excessive false alarms may lead to low response rates to the alarms [9] . Bridi, da Silva et al. (2014)conducted a quantitative, descriptive study at the coronary care unit in InstitutoNacional de CardiologiaHospitalin Rio de Janeiro state in Brazil. Eighty eight patients were monitored; divided into 49 patients monitored within day shift, and 39 patients within night shift. The data were collected by monitoring "out of duty" nurse and record each observation related to responding to alarms. The data were collected over a total of four month, March to June 2012, with total observation 20 hours in day shift and 20 hours in night shift, with no standard protocol for monitoring. Everyone in the research team was authorized to adjust the alarm limits according to his/her own experiences. The machines used in the unit were basically infusion pumps, mechanical ventilators, cardiac monitor, and intraaortic balloon pump. The results of the study indicated that there was a total of 227 alarms during the observation time, 106 of which are on day time, out of them 68 alarms were not responded for $(64.15 \%)$. On the other hand, 77 alarms not answered within night shift $(63.64 \%)$. In $60 \%$ of the alarms the response was delayed to longer than 10 minutes, which was considered as no response to alarms. The median response to answered alarms was 4 minutes and 54 seconds in the day shift and in night shift was 4 minutes and 55 seconds. In conclusion, the findings of the study supported other studies' findings in that there is a delay and/or lack of proper response to clinical alarms; which has a serious impact on patient safety [5].

\subsection{Desensitization to Alarms:}

In order to understand the term 'desensitization' to clinical alarms, we need to know the meaning of specificity and sensitivity. Specificity means the ability of the alarms to alert for a medical condition that requires attention from the care provider [31]. While sensitivity is the ability of the alarms sound to alert when it exceeds the threshold. This means they are determinants for the equipment that come with pre-set alarm defaults that continue to activate alarms till anyone from care provider respond appropriately to the alarm. However, care providers who desire alarm specificity (where alarms are triggered by real events) have suggested having the ability to personalize alarms to patient conditions that warrant closer monitoring [31] .Present sensitivity of alarms is close to $100 \%$, but the specificity, which is necessary for healthcare staff, cannot be determined. One of the most important challenges to decrease the number of false alarms is determining which alarms are clinically significant. Alarm design that is both sensitive and specific is missing due to the opposing needs of alarm manufacturers and clinicians [31] .

Graham and Cvach (2010)conducted a pilot study from January 2006 through June 2007, on a 15-bed Medical progressive care unit (MPCU) with 30 nurses. The quality improvement project was designed from nursing concerns regarding excessive numbers of alarms and their effect on patient safety. The project begins with a hospital cardiac monitoring protocol that required nurses to individualize alarm setting to their patient needs at the beginning of the shift. Post-intervention alarm frequency was compared to baseline frequency. The total number of alarms was reduced from 16,953 to 9,647 showing a $43 \%$ reduction in critical monitor alarms within 17 month. Post-test survey results showed an $83 \%$ changed alarm setting when a patient's hemodynamic changed and $78 \%$ changed setting at the beginning of their shift. Nurse's perception of noise levels were reduced from 4.0 to 3.5 . Noise of monitor alarm was reduced from 3.1 to 2.9 reflecting an overall satisfactory reduction of noise [15].

Graham and Cvach in 2010, study showed that while alarms can present helpful and often lifesaving information, sensitivity related to equipment monitoring makes it necessary to individualize alarm settings. It prevents from delayed response ordesensitization practiced by nurses who are exposed to alarm noises that may be clinically false, but affected by the alarm's sensitivity [15]. The study indicates that a multidisciplinary team, involving a nursing leaders, register nurses, and biomedical engineers representing critical care units is necessary to initiate awareness of the importance of clinical alarm management and prioritizing alarm settings. 


\subsection{Non-Actionable alarms and False Alarms:}

Sendelbach and Funk (2013) described that the false alarm occurs when the monitor system issue the alarm not valid and excessive numbers from this alarms may lead to alarm fatigue [11]. While the nonactionable alarms are those true alarms that have no immediate action or attention of healthcare providers.

Edworthy (2013)conducted a review to clarify that false alarms not acceptable by using the aims: overview of main issue which is important in designing the alarm systems, and equality by standardization the audible alarm system in medical field, discuss how the false alarms lead to negative effects, and the need to apply evidence base practice in designing medical alarms [11].

The philosophy of alarm design focused on the alarm as a whole; to decrease the mortality rates, we need to consider preventing the alarm fatigue. So the designer continues development to alarm system to make them as safe as possible. Therefore, when the nurses encounter false alarm this leads to missing one of the important critical alarm effect in patient safety. In this review, it was noticed that the monitor design contributes to the high rates of false alarm, which, in turn, lead to alarm fatigue.

Edworthy talk over that the excessive invariant alarm sound mostly impaired nurses' management of important alarms. The auditory concepts differ by class of sound. The designers of alarms failed to consider the whole environment when they developed the alarm settings, which made it difficult for nurses to overcome challenges of recognizing and interpreting alarms. Although the philosophy of alarm intended to design alarm specific to decrease the excessive of alarm sound [30] .

Edworthy (2013) noticed that arrangements went for bringing down false alert rates are promising. The healthcare providers, especially the nurses, ability to individualize patient status related alarm has appeared to decrease false alert rates [11]. Alarm choice and the aim for use must be included in the discussion between the nurses and alarm designer to find out proper strategies when developing the system. In spite of the importance of having standard process for designing alarms, but each machine need to have specific setting. In this manner, the nurses' attitudes of clinical alarm can be effective for process improvement, and in the development of new policies to decrease false alarms.

The non-actionable alarm also contribute to alarm fatigue phenomenon [21] .

The high numbers of alarms that are clinically true but not relevant to patient's case, when there is no need for any intervention from healthcare providers, should be eliminated as they can lead to alarm fatigue [36] .

Bonafide, Lin et al.(2015)conducted an observational study about the relationship between nurses exposed to high rate of non-actionable physiological monitor alarms and the time of responding to critical alarms. 36 nurses were observed in the pediatric intensive care unit in the Children Hospital of Philadelphia/Pennsylvania, for 210 hours with 5070 alarms. The author found $87.1 \%$ of PICU and $99 \%$ of medical ward alarms were not actionable, and if non-actionable alarms increase they lead to delay in response time. The researcher concluded that the non-actionable alarms have direct effect and lead to unsafe practice, and they decrease time of nursing response, all of them lead to enhance patient safety [2].

\subsection{Impact of Alarm Fatigue on Patient Safety}

From August 2005 to January 2006 the American College of Health Care Technology Foundation (HTF) conduct the study towered alarm issue after data collected from 1327 care givers and engineers by used clinical alarm survey tool, and the recommended from result of survey to enhance the impact of clinical alarm on patient safety, and how many contributing false alarms on response staff to true alarms [12] . Also the ECRI committee reviews the result of the clinical alarm survey that the most importance result of the survey was high level of noise alarms and false alarms. Also, the False alarms got the large part from ICCU nursing time from bedside work. And if false alarms significant decline, the level of clinical practice towered staff should be improved [12] .

\section{Conclusion}

The literature review showed that the alarm fatigue resulted in lack of response of health care providers due to excessive numbers of alarms, which lead to desensitization and sensory overload, and multiple factor such as non-actionable alarms and false alarms. Biomedical engineers make every effort for sensitivity, while healthcare providers need to understand specificity as it is important while taking care of patient in critical care units. Ongoing evaluation of nurses' attitudes related to alarm fatigue will help the hospitals to make and develop standard policies and education related to clinical alarms, which is considered as a major requirement of different accreditation systems.

\section{Acknowledgements}

The authors acknowledge the support from the alkhalidi hospital for the financial support granted to this research and everyone assist in this project. 


\section{References}

[1]. $\quad$ Authority, P. P. S. (2008). "Alarm interventions during medical telemetry monitoring: a failure mode and effects analysis." Pennsylvania, PA: Pennsylvania Patient Safety Advisory.

[2]. Bliss, J. P., et al. (1995). "Human probability matching behaviour in response to alarms of varying reliability." Ergonomics38(11): 2300-2312.

[3]. Bonafide, C. P., et al. (2015). "Association between exposure to nonactionable physiologic monitor alarms and response time in a children's hospital." Journal of hospital medicine.

[4]. Baird, Kevin Michael (2015). " Examining the Problem of Alarm Fatigue: An Analysis of Intensive Care Nurse's Attitudes Related to Clinical Alarm Management"

[5]. Bridi, A. C., et al. (2014). "Reaction time of a health care team to monitoring alarms in the intensive care unit: implications for the safety of seriously ill patients." Revista Brasileira de Terapia Intensiva26(1): 28-35.

[6]. Bustamante, E., et al. (2007). "Effects of varying the threshold of alarm systems and workload on human performance." Ergonomics50(7): 1127-1147.

[7]. Chambrin, M.-C., et al. (1999). "Multicentric study of monitoring alarms in the adult intensive care unit (ICU): a descriptive analysis." Intensive care medicine25(12): 1360-1366.

[8]. Chopra, V. and L. F. McMahon (2014). "Redesigning hospital alarms for patient safety: alarmed and potentially dangerous." JAMA311(12): 1199-1200.

[9]. Cvach, M. (2012). "Monitor alarm fatigue: an integrative review." Biomedical Instrumentation \& Technology46(4): $268-277$.

[10]. ECRI (2015). "ECRI Institute Top 10 Health Technology Hazards Report for 2014". http://www.visimobile.com/wpcontent/uploads/2013/12/6C-5-Top-10-Health-Tech-Hazards-2014-\%E2\%80\%93-ECRI.pdf. Accessed 25 April 2016.

[11]. Edworthy, J. (2013). "Medical audible alarms: a review." Journal of the American Medical Informatics Association 20(3): 584-589.

[12]. Force, C. (2007). "Impact of clinical alarms on patient safety: a report from the American College of Clinical Engineering Healthcare Technology Foundation." Journal of Clinical Engineering 32: 22-33.

[13]. Funk, M., et al. (2014). "Attitudes and practices related to clinical alarms." American Journal of Critical Care23(3): e9-e18

[14]. Getty, D. J., et al. (1995). "System operator response to warnings of danger: A laboratory investigation of the effects of the predictive value of a warning on human response time." Journal of Experimental Psychology: Applied1(1): 19.

[15]. Graham, K. C. and M. Cvach (2010). "Monitor alarm fatigue: standardizing use of physiological monitors and decreasing noise alarms." American Journal of Critical Care19(1): 28-34.

[16]. Henderson, Y. (2008). "Delivering oxygen therapy to acutely breathless adults." Nursing Standard 22(35): 46 Henriksen, K., et al. (2005). "Best Practices for Medical Technology Management: A US Air Force-ECRI Collaboration."

[17]. Horkan, A. M. (2014). "Alarm fatigue and patient safety." Nephrology Nursing Journal41(1): 83.

[18]. Inokuchi, R., et al. (2013). "The proportion of clinically relevant alarms decreases as patient clinical severity decreases in intensive care units: a pilot study." BMJ open3(9): e003354.

[19]. Keller, J. P. (2012). "Clinical alarm hazards: a "top ten" health technology safety concern." Journal of electrocardiology45(6): 588591.

[20]. Keller, J. P., et al. (2011). "Why clinical alarms are a'top ten'hazard: how you can help reduce the risk." Biomedical Instrumentation \& Technology45(s1): 17-23.

[21]. Kerr, J. and B. Hayes (1983). "An "alarming" situation in the intensive therapy unit." Intensive care medicine9(3): 103-104

[22]. Kestin, I., et al. (1988). "Auditory alarms during anesthesia monitoring." Anesthesiology69(1): 106.

[23]. Khaled Sowan A., Tarriela A.F., Gomez T.M., Reed C.C. \& Rapp K.M. (2015). "Nurses' Perceptions and Practices Toward Clinical Alarms in a Transplant Cardiac Intensive Care Unit: Exploring Key Issues Leading to Alarm Fatigue". JMIR Human Factors 2(1): e3.

[24]. Korniewicz, D. M., et al. (2008). "A national online survey on the effectiveness of clinical alarms." American Journal of Critical Care 17(1): 36-41.[26]Lawless, S. T. (1994). "Crying wolf: false alarms in a pediatric intensive care unit." Critical care medicine22(6): 981-985.

[25]. Meredith, C. and J. Edworthy (1995). Sources of confusion in intensive therapy unit alarms. Human factors in alarm design, Taylor \& Francis, Inc.

[26]. Polit, D. F. and C. T. Beck (2010). Nursing research: Principles and methods, Lippincott Williams \& Wilkins.

[27]. Saltzman (2013), W. Investigation: dangers of medical recovery rooms.

[28]. Sendelbach, S. and M. Funk (2013). "Alarm fatigue: a patient safety concern." AACN advanced critical care24(4): 378-386.

[29]. Siebig, S., et al. (2010). "Intensive care unit alarms - How many do we need?*." Critical care medicine38(2): 451-456.

[30]. Solet, J. M. and P. R. Barach (2012). "Managing alarm fatigue in cardiac care." Progress in Pediatric Cardiology33(1): 85-90.

[31]. Stafford, T. (1982). "Whither monitoring?" Critical care medicine10(11): 792-795.

[32]. The Joint Commission. Sentinel event alerts: Medical device alarm safety in hospitals.: April8,issue 50; 2013. URL:http://www.jointcommission.org/assets/1/18/SEA_50_alarms_4_5_13_FINAL1.PDF [accessed 2015-01-01]

[33]. Urden, L. D., et al. (2013). Critical Care Nursing, Diagnosis and Management, 7: Critical Care Nursing, Elsevier Health Sciences

[34]. Weaver, J. M. (2013). "Alarm Fatigue Can Decrease the Safety of Dental Office Sedation and Anesthesia." Anesthesia progress(3): 93-94.

[35]. Welch, J. (2011). "An evidence-based approach to reduce noise alarms and alarm fatigue." Biomedical Instrumentation \& Technology45(s1): 46-52. 